\title{
Archives
}

\section{Forces vives oubliées de la culture québécoise : les archives religieuses}

\author{
Comité des archives du Conseil du patrimoine religieux du Québec, \\ Regroupement des archivistes religieux du Québec et Table de concertation \\ des archives religieuses de la région de Montréal (DAVID BUREAU, STEPHANE \\ COMEAU, MARIE-PIERRE COURCHESNE, HELENE ÉLEMENT, MARC LACASSE, \\ STEPHAN MARTEL, ELAINE SIROIS)
}

Volume 47, numéro 2, 2018

MÉMOIRES SUR LE RENOUVELLEMENT DE LA POLITIQUE CULTURELLE DU QUÉBEC (2016)

URI : https://id.erudit.org/iderudit/1045169ar

DOI : https://doi.org/10.7202/1045169ar

Aller au sommaire du numéro

Éditeur(s)

Association des archivistes du Québec (AAQ)

ISSN

0044-9423 (imprimé)

2369-9256 (numérique)

Découvrir la revue

Citer cet article

Comité des archives du Conseil du patrimoine religieux du Québec,

Regroupement des archivistes religieux du Québec et Table de concertation des archives religieuses de la région de Montréal (2018). Forces vives oubliées de la culture québécoise : les archives religieuses. Archives, 47(2), 59-75.

https://doi.org/10.7202/1045169ar

\section{Résumé de l'article}

Le patrimoine religieux a une place importante dans l'histoire du Québec ; ses archives, qui en constituent une pièce maîtresse, reflètent des facettes de la société comme l'éducation, la santé, les services sociaux, la diversité culturelle, le réseau de la francophonie et la culture autochtone. Cependant, plusieurs archives religieuses sont menacées de disparition en raison du vieillissement et de la disparition des communautés religieuses, qui en sont les gardiennes. Leur vulnérabilité s'explique aussi par des conditions de conservation inadéquates et un sous-financement chronique de la part du gouvernement.

Dans ce mémoire, plusieurs solutions pour sauver les archives sont proposées, comme la création de centres régionaux par la mise en commun des ressources des diverses communautés. Par exemple, à Montréal, où le problème est criant, la mise sur pied d'un centre de patrimoine religieux est en branle, bien que plusieurs problèmes de financement freinent le projet. C'est à travers une vision globale du patrimoine religieux que la situation pourra s'améliorer. À cela, on peut ajouter une aide à l'utilisation et à la diffusion des archives, une aide à l'embauche de main-d'oeuvre et de stagiaires, des partenariats avec les municipalités et un aménagement du territoire favorisant la conservation du patrimoine.
Tous droits réservés @ Association des archivistes du Québec (AAQ), 2018
Ce document est protégé par la loi sur le droit d'auteur. L’utilisation des services d'Érudit (y compris la reproduction) est assujettie à sa politique d'utilisation que vous pouvez consulter en ligne. 


\title{
MÉMOIRE
}

DOSSIER

SPÉCIAL

\section{Forces vives oubliées de la culture québécoise: les archives religieuses}

\begin{abstract}
COMITÉ DES ARCHIVES DU CONSEIL DU PATRIMOINE RELIGIEUX DU QUÉBEC, REGROUPEMENT DES ARCHIVISTES RELIGIEUX DU QUÉBEC ET TABLE DE CONCERTATION DES ARCHIVES RELIGIEUSES DE LA RÉGION DE MONTRÉAL
\end{abstract}

\section{DAVID BUREAU}

Archiviste, Oratoire Saint-Joseph du Mont-Royal

\section{STEPHANE COMEAU}

Adjoint du chancelier et archiviste, Archevêché de Montréal

\section{MARIE-PIERRE COURCHESNE}

Archiviste, Service des archives de l'Administration générale des Religieuses Hospitalières de Saint-Joseph

\section{HELENE ÉLEMENT}

Responsable des archives, Congrégation des Sœurs de Sainte-Anne 


\section{MARC LACASSE}

Archiviste et coordonnateur du département des archives, Univers culturel de Saint-Sulpice

\section{STEPHAN MARTEL}

Historien, gestionnaire du Centre de documentation et archives, Musée Marguerite-Bourgeoys

\section{ELAINE SIROIS}

Archiviste et directrice, Archives Deschâtelets-NDC des missionnaires Oblats de Marie Immaculée

\section{PRÉSENTATION DES ORGANISMES SIGNATAIRES}

\section{Le Conseil du patrimoine religieux du Québec (CPRQ), Comité des archives}

C'est en 2006 que le comité des archives voit le jour au sein du Conseil du patrimoine religieux du Québec (CPRQ). Fondé en 1995, le CPRQ est un organisme à but non lucratif qui œuvre à l'échelle du Québec avec pour mission de soutenir et de promouvoir la conservation et la mise en valeur du patrimoine religieux québécois. Le comité des archives témoigne du souci du CPRQ d'inclure le patrimoine archivistique dans son champ d'intervention. Ce comité est composé d'archivistes de communautés religieuses féminines, masculines, d'archivistes diocésains (dont le diocèse anglican) de deux historiens et de collaborateurs ponctuels. II a pour but d'encourager les représentants de différentes traditions religieuses et leurs communautés religieuses à assurer la pérennité de leurs archives, afin d'en garantir la valeur de témoignage pour les générations à venir. Le comité vise entre autres à: 1 . Promouvoir les archives religieuses en sensibilisant la population et les décideurs à l'importance de ce patrimoine; 2 . Soutenir toute initiative émanant du milieu des archivistes religieux qui concourent à remplir sa mission; 3. Favoriser les échanges entre les différents intervenants du patrimoine. De 2012 à 2016, le comité des archives a tenu quatre Journées des archives religieuses rassemblant un total de près 
de 500 intervenants(es) afin d'aborder des sujets touchant la pérennité des archives religieuses et leur mise en valeur. En 2016, anticipant les besoins qui vont toucher les détenteurs d'archives, le comité a rédigé un guide de déménagement de dépôt d'archives.

\section{Le Regroupement des archivistes religieux du Québec (RAR)}

Créé en 1978 et incorporé en 1991, le Regroupement des archivistes religieux est une association au service des archivistes qui œuvrent dans les congrégations religieuses, les chancelleries diocésaines, les fabriques de paroisses, les instituts séculiers, les ordres et les sociétés religieuses. Elle offre à ces professionnels de la mémoire, désireux de s'entraider, un soutien dans l'accomplissement de leur tâche et un lieu de formation et d'échange sur tout sujet relatif à la préservation, la diffusion et l'avenir des archives ecclésiastiques dont ils sont les responsables.

\section{La Table de concertation des archives religieuses de la région de Montréal (TCARM)}

Fondée en janvier 2014, la Table de concertation des archives religieuses de la région de Montréal est une initiative du Regroupement des archivistes religieux (RAR) et de congrégations religieuses. Elle regroupe une vingtaine de communautés membres, actives ou ayant été actives à Montréal. Dans le contexte actuel où la majorité des communautés religieuses subissent une décroissance du nombre de leurs membres et doivent se départir de plusieurs de leurs biens, la TCARM a pour objectif principal de mettre sur pied un Centre du patrimoine religieux à Montréal, qui aura pour objet la préservation et la diffusion des archives, des publications, des biens mobiliers et des œuvres d'art témoignant de l'histoire et de l'importance du fait religieux pour l'histoire du Québec. Ce Centre vise à offrir des solutions pérennes afin d'assurer l'avenir de cet héritage et sa mise en valeur. II aura aussi pour mission d'être un pôle touristique, historique, éducatif et culturel. Le comité exécutif travaille actuellement à doter la TCARM d'un statut juridique, à trouver du financement et un lieu pour accueillir ce futur Centre. En parallèle, il s'active aussi à mettre en place des activités de réseautage et de sensibilisation afin de mettre en valeur le projet et à en faciliter la mise en place. 


\section{PRÉLUDE}

Depuis la publication du mémoire sur l'avenir des archives religieuses, il s'est écoulé plus d'une année et le projet de la Table de concertation des archives religieuses de Montréal (TCARM) a pris son envol. Déjà, le sigle TCARM est délaissé pour s'adapter à sa nouvelle réalité, plus concrète, celle du Centre d'archives et de patrimoine religieux de Montréal (CAPREM). Les deux termes se réfèrent à la même entité, à la seule exception que le CAPREM reflète un certain degré de maturité atteint par le projet.

Dès 2016, le CAPREM a suscité l'intérêt de la Ville de Montréal. Depuis, un comité bilatéral formé des membres du comité exécutif du CAPREM, de représentants de la Ville de Montréal, d'un représentant de Bibliothèque et Archives nationales du Québec (BAnQ) et du ministère de la Culture et des Communications (MCC) du gouvernement du Québec a été mis en place. En 2017, I'achat du couvent des Religieuses hospitalières de Saint-Joseph par la Ville de Montréal ouvre une opportunité au CAPREM de faire partie du projet. Nommé Cité des Hospitalières, la thématique développée pour le site sera celle du «vivre ensemble». Cette proposition a été accueillie avec enthousiasme par les communautés religieuses et les organismes membres du CAPREM.

Récemment, le CAPREM a engagé son premier directeur en la personne de Monsieur Simon Bissonnette. Ce dernier cumule plus de quinze années d'expérience en direction au sein de moyennes et grandes organisations. II est par ailleurs l'artisan de la restauration et l'ouverture au public de la maison Félix-Leclerc à Vaudreuil. Son premier mandat au CAPREM sera de doter l'organisme d'une existence juridique. Un premier pas vers l'existence officielle de l'organisme pour sa pérennité et celle du patrimoine religieux à sauvegarder et à transmettre.

Pour d'autres informations sur le CAPREM, veuillez contacter Monsieur Simon Bissonnette au (514) 397-9415 ou par courriel: sbissonnette@ tcarm.org

\section{INTRODUCTION}

Le présent mémoire est le fruit des efforts d'archivistes et d'historiens professionnels qui, au-delà de leurs activités quotidiennes dans leurs 
institutions respectives, s'impliquent bénévolement au sein de leur milieu associatif et trouvent du temps pour promouvoir la conservation et la mise en valeur du patrimoine archivistique.

\section{LA RICHESSE DES ARCHIVES RELIGIEUSES}

Le milieu des archives religieuses, représenté par les associations œuvrant pour leur sauvegarde et leur diffusion, est particulièrement interpellé par le renouvellement de la politique culturelle du gouvernement du Québec. Chroniquement sous-financées, souvent en danger ou déjà perdues, les archives religieuses revendiquent la reconnaissance de leur rôle comme pilier de la culture québécoise.

Il est impossible de soustraire le fait religieux de l'histoire québécoise. Des découvreurs et colons, essentiellement des chrétiens catholiques, sont venus en Nouvelle-France, pour s'y établir. Très tôt, des communautés religieuses françaises se sont implantées dans la colonie. Les Récollets, Jésuites, Sulpiciens, Ursulines, Hospitalières de Saint-Joseph et les Augustines, animés par une mission d'évangélisation, ont bâti des écoles et des hôpitaux ${ }^{1}$. Certains ordres religieux ont leur origine en terre canadienne, comme les sœurs de la Congrégation de Notre-Dame et les Sœurs de la Charité de Montréal (Sœurs Grises). La forte influence de l'Église catholique en Nouvelle-France s'explique également par l'édit de Fontainebleau, que proclama Louis XIV en 1685, qui interdisait aux non-catholiques de s'établir dans la colonie. En dépit de la Conquête britannique, la religion catholique s'est maintenue, puis a continué à croître en notre province. C'est pourquoi les archives religieuses du Québec sont surtout catholiques, bien qu'elles s'enrichissent depuis la conquête de la Nouvelle-France par la Grande-Bretagne de l'apport des traditions anglicanes, protestantes, orthodoxes, juives, et, plus récemment, musulmanes, sans oublier les traces des traditions autochtones.

Les organismes religieux créateurs d'archives sont divers. II y a notamment:

- Les communautés religieuses où les membres sont réunis selon un rythme structuré par la prière et les différents services communs². Chaque communauté a son propre charisme et sa propre mission dans l'Église; 
- Les paroisses, subdivisions territoriales relevant d'un diocèse, où une communauté précise de fidèles bénéficie des services d'un curé;

- Les diocèses, circonscriptions ecclésiastiques dirigées par un évêque ;

- Les mouvements, œuvres sociales et autres organismes ayant eu une dimension religieuse à leur origine et dans leur mission.

Les archives religieuses sont bien plus que de simples chroniques à citer dans les livres d'histoire, dans les festivals et les commémorations. Ces archives sont aussi indispensables afin de documenter et de contextualiser les autres types de patrimoines religieux.

Les patrimoines immobiliers et immatériels ont fait l'objet d'une grande attention de la part des autorités en terme de subventions ou d'actions concrètes de préservation ces dernières années. Toutefois, que serait une église sans les plans de celle-ci, soigneusement conservés au fil des décennies et des documents expliquant les raisons de cette construction? Comment restaurer des biens immobiliers sans photographies et documents textuels expliquant leur utilisation au fil du temps? Comment saisir toute la signification des objets de culte sans comprendre les croyances et les usages qui y sont reliés? Les archives constituent une pièce maîtresse du patrimoine religieux et culturel du Québec et méritent une attention primordiale à I'heure où beaucoup d'entre elles sont menacées de disparition due à des conditions de conservation inadéquates et au manque de financement.

D'un point de vue beaucoup plus large, les archives ecclésiales permettent non seulement de documenter I'histoire des communautés religieuses et des institutions des différentes églises, mais aussi l'histoire de la société québécoise dans son ensemble. Tous citoyens et citoyennes peuvent se sentir interpellés par le patrimoine religieux puisqu'il se trouve à l'origine d'un grand nombre de nos institutions contemporaines, notamment des milieux éducatif, hospitalier, culturel, etc. Notons, par exemple, les liens historiques avec les autres pays de la Francophonie, dont nous conservons les traces dans les archives religieuses québécoises. Plusieurs communautés religieuses proviennent de ces pays ou encore ont envoyé des missionnaires dans d'autres pays de la Francophonie. La diffusion des archives qui en témoignent suscite l'intérêt des citoyens et permet ainsi d'accroître la place de la culture du Québec au sein du réseau de la Francophonie. Par ailleurs, les archives religieuses du Québec 
sont avant tout francophones. Assurer leur préservation et encourager leur diffusion revient incidemment à promouvoir et à préserver la langue française, tant au Québec que dans la Francophonie. Les archives religieuses contiennent des éléments historiques incontournables sur I'origine du français au Québec et sur son évolution. Elles sont aussi porteuses de l'histoire de l'évolution de l'enseignement du français et de l'usage de la langue dans la province.

De plus, les archives religieuses reflètent la diversité culturelle québécoise à travers son histoire, car de nombreuses communautés ont œuvré auprès des cultures d'où proviennent les immigrants qui s'installent au Québec. Les innombrables photographies, documents sonores et audiovisuels, les récits de voyage des missionnaires décrivant les us et coutumes de ces pays, sans compter les artéfacts apportés par les missionnaires, constituent des témoignages éloquents et souvent rares de ces cultures. Aussi, depuis plus d'un siècle, les diocèses du Québec ont créé des «missions» locales, pourvues d'églises et de personnel pastoral, destinées à accueillir les divers regroupements laïques ethniques et culturels qui sont catholiques. Cette longue histoire des communautés et des diocèses mérite d'être davantage diffusée pour favoriser l'intégration et la participation à la vie culturelle des personnes issues de l'immigration. Les religieux et religieuses ont aussi côtoyé les autochtones depuis les débuts de la Nouvelle-France jusqu'à aujourd'hui. Plusieurs missionnaires ont appris les langues autochtones et développé des dictionnaires et des grammaires qui sont actuellement conservés dans les archives des communautés religieuses. Certains centres d'archives contiennent aussi des enregistrements sonores et audiovisuels de chants ancestraux. Une meilleure diffusion de ces documents pourrait être utile aux communautés autochtones dans leurs efforts de réappropriation de leurs langues.

À considérer toute cette richesse des archives religieuses du Québec, il ne faut surtout pas négliger qu'elles sont un legs que les organismes religieux désirent ardemment partager avec les citoyens et citoyennes. Ces organismes souhaitent, sans vouloir en faire un profit pécuniaire, que leur patrimoine et leur histoire soient reconnus à leur juste valeur et diffusés à grande échelle. 


\section{2. ÉTAT DE LA SITUATION}

Dans un contexte de décroissance et de vieillissement des membres des communautés religieuses, l'intégrité des archives est parfois mise en péril. En effet, les responsables religieux doivent assurer avant tout le bien-être de leurs membres, vendre les propriétés désormais trop grandes pour leurs besoins et, parfois même, se relocaliser. Les profits de la vente des bâtiments servent en priorité à subvenir aux besoins essentiels des membres des communautés. Quant aux archives, plusieurs communautés doivent leur trouver un nouveau lieu de conservation. Plusieurs communautés se sentent démunies, peu outillées pour mener à bien le déménagement d'un service d'archives. D'autres ont peu de moyens financiers pour embaucher des responsables de leurs patrimoines archivistique, documentaire et muséal. La décroissance et le vieillissement des membres des communautés religieuses provoquent un état d'urgence qu'un sondage, réalisé par la Table de concertation des archives religieuses de la région de Montréal (TCARM) auprès de ses membres, illustre clairement. Le sondage révèle que d'ici 10 ans, douze des treize communautés montréalaises approchées ne pourront plus assurer la conservation de leurs archives. La période d'autonomie de conservation du patrimoine religieux de ces communautés a été estimée ${ }^{3}$ à:

- 3 ans et moins pour 3 communautés;

- 3 ans à 8 ans pour 4 communautés;

- 5 à 10 ans pour 5 communautés;

- 10 à 15 ans pour 1 communauté.

Une solution doit rapidement être trouvée pour certaines communautés de Montréal et d'autres régions du Québec. Cette situation d'urgence n'est pas surprenante, étant donné que, depuis longtemps, il n'existe pratiquement aucune source de financement pérenne dédiée spécifiquement aux archives religieuses. Déjà en 2005, un mémoire de l'Association des archivistes du Québec faisait mention du sousfinancement grave du patrimoine archivistique religieux. Le financement de l'État pour le soutien aux organismes chargés des archives religieuses ne représentait alors qu'un maigre 1,5\% du montant total consacré aux autres formes de patrimoine ${ }^{4}$. Les ressources limitées offertes par Bibliothèque et Archives nationales du Québec (BAnQ), laissent croire 
à un désengagement à répondre aux besoins d'organismes privés détenteurs de patrimoine archivistique, puisqu'aucun montant n'est dédié spécifiquement aux archives religieuses. Les programmes de soutien financier pour le traitement des archives sont régulièrement réduits et visent des objectifs limités à très court terme. Quant au Conseil du patrimoine religieux du Québec (CPRQ), il faudrait lui donner les moyens de soutenir son comité des archives, qui ne bénéficie d'aucune enveloppe budgétaire pour des projets particuliers. Pour réaliser ses activités, le comité des archives doit procéder par recherche de commanditaires et de subventions, s'appuyer sur des frais d'inscription et la collaboration gracieuse d'institutions pour la gratuité de locaux.

Le Regroupement des archivistes religieux du Québec (RAR) s'est efforcé, dès 1998, à trouver des solutions viables pour la sauvegarde des archives religieuses. Parmi ses récents efforts, le RAR a encouragé, et soutenu, la création de tables de concertation des archives religieuses dans plusieurs régions du Québec.

Par exemple, la Table de concertation des archives religieuses de la région de Montréal (TCARM), fondée en janvier 2014, regroupe une vingtaine de communautés membres, actives ou ayant été actives à Montréal. La TCARM a pour objectif principal de mettre sur pied un Centre du patrimoine religieux à Montréal. Ce Centre aura pour objet la préservation et la diffusion des archives, des publications, des biens mobiliers et des œuvres d'art témoignant de l'histoire et de l'importance du fait religieux pour I'histoire du Québec. II vise à offrir des solutions pérennes afin d'assurer l'avenir de cet héritage et sa mise en valeur. La création d'un tel centre d'archives est un défi de taille. Pour en comprendre l'envergure, le RAR a commandé, en cours d'année 20152016, une analyse de préfaisabilité pour ce type de projet. Cette analyse, que I'on retrouve à l'annexe 1 de ce mémoire, contient, entre autres, des recommandations pour la création d'une fondation, des suggestions pour le financement du projet ainsi qu'une analyse des risques et des facteurs de succès. II est à noter que la recherche d'un lieu pour le Centre est une des tâches les plus ardues. D'un côté, les ressources humaines et financières pour mettre en place le projet sont limitées ou inexistantes. De l'autre, certaines communautés, avant de se joindre et de contribuer à la TCARM, attendent qu'un lieu soit déjà choisi pour le projet. Ensuite, il faut financer la construction même du centre. 
Par exemple, dans une deuxième analyse commandée par le RAR, que I'on retrouve à l'annexe 2 de ce mémoire, une firme d'architectes a estimé à 3900000 \$ le coût de la construction d'un petit centre d'archives qui pourrait satisfaire, minimalement, les besoins actuellement urgents pour la sauvegarde des archives religieuses de la région de Montréal. Selon l'analyse, la construction d'un centre pouvant satisfaire les besoins de conservation à plus long terme, c'est-à-dire les besoins de conservation pour beaucoup plus d'organismes religieux de Montréal et d'ailleurs, et pouvant offrir tous les services de diffusion d'archives envisagés, pourrait coûter jusqu'à 10600000 \$. Il faut ajouter à ces coûts, le coût d'achat du terrain et les frais annuels pour la gestion du centre.

De la préservation du contenu culturel que sont les archives religieuses, jusqu'à sa diffusion et son impact sur la participation citoyenne, les centres d'archives religieuses s'inscrivent certainement dans la chaîne culturelle québécoise. Cependant, le manque de ressources fait en sorte que cette chaîne est fragilisée dès ses débuts. Bien que les centres d'archives religieuses visent à accorder aux archives un traitement archivistique selon les normes établies, les ressources actuelles ne permettent pas aux archives religieuses d'être diffusées et exploitées à leur juste valeur. Les centres d'archives religieuses peinent à répondre aux besoins en conservation, en traitement, en accessibilité et en mise en valeur de leurs archives. Il va sans dire, conséquemment, que le réseau d'équipements culturels (musées, bibliothèques, salles de spectacles, etc.) des centres d'archives religieuses est loin d'être aussi bien établi que les autres réseaux.

\section{RECOMMANDATIONS}

\subsection{Une vision globale du patrimoine religieux}

La situation du milieu archivistique québécois, et particulièrement celui du milieu archivistique religieux, gagnerait à être mieux documentée. ॥ serait nécessaire que la première grande enquête qui a été menée en 2005 par l'Institut de la statistique du Québec ${ }^{5}$ soit reprise à intervalles réguliers afin d'obtenir une image à jour du milieu archivistique au Québec et de son évolution. II serait même souhaitable que cette enquête soit élargie, en y ajoutant un volet permettant de mieux connaître les sources de financement et le fonctionnement des services d'archives dans la province. Plus spécifiquement, un observatoire pour le patrimoine religieux 
devrait aussi être mis sur pied, car une vision d'ensemble, à l'échelle de la province, est nécessaire afin de suivre l'évolution de la situation au Québec. Cette vision d'ensemble offrirait la possibilité d'une gestion plus intégrée de notre patrimoine religieux au Québec. Le développement de cette vision d'ensemble permettrait aussi de mieux comprendre les réalités et les besoins de chacune des régions. Il est nécessaire que nous connaissions l'état de la situation des communautés religieuses les plus à risque et que nous puissions les soutenir avant qu'il ne soit trop tard. À l'heure actuelle, de nombreux fonds d'archives religieuses sont déjà perdus et plusieurs sont à risque de l'être.

\subsection{Aide à I'utilisation et à la diffusion des archives}

Notons ici quelques exemples où l'aide à l'utilisation et à la diffusion des archives religieuses aurait des impacts significatifs pour le Québec.

Au cours des dernières années, de nombreux projets de restauration de bâtiments religieux ont nécessité des recherches dans les archives institutionnelles. À certaines occasions - et particulièrement pour les interventions sur des bâtiments classés - on a tenu pour acquis que l'expertise du personnel du service d'archives (en recherche documentaire, analyse et synthèse documentaires, reproductions d'archives textuelles, photographiques, devis et plans, etc.) pouvait se réaliser sur demande et relevait de la plus complète gratuité. Considérant la complexité de tels dossiers en planification de travaux, il devrait être requis que tout projet de restauration tienne compte d'un volet « documentation et recherche», doté d'un budget conséquent afin de produire un «rapport d'état patrimonial » comprenant bilans historiques, architecturaux, artistiques, archéologiques, ethnographiques, etc., se rapportant à l'évolution et les usages du bâtiment au fil de son existence et à son environnement. De tels rapports demeurent utiles sur bien des aspects, non seulement dans leur réutilisation lors d'interventions subséquentes sur l'édifice, mais également à titre de modèles comparatifs, de bilan d'expériences ou document de référence pour soutenir la planification d'interventions sur des bâtiments comparables dans d'autres régions.

Encourager les centres d'archives religieux à faire de la diffusion sur Internet serait une façon simple de favoriser, pour le gouvernement du Québec, la visibilité des contenus culturels québécois francophones. 
Au niveau local, soutenir la diffusion favoriserait la participation citoyenne des Québécois. Les citoyens et citoyennes, étant mieux sensibilisés par l'histoire religieuse du Québec, se sentiraient davantage responsables à l'égard de ce patrimoine. Tout cela pourrait améliorer les conditions socioéconomiques de ceux qui travaillent en ce moment dans le milieu religieux, tels les intervenants en pastorale, les animateurs, les conférenciers, les chercheurs, etc. Ces travailleurs pourraient alors contribuer davantage à la création de nouveaux documents alimentant les archives religieuses. Ainsi, I'aide gouvernementale initiale pour la diffusion peut créer un cercle vertueux qui favoriserait, par la suite, I'autofinancement.

\subsection{Main-d'œuvre et stages}

Les collaborations avec des instances publiques et privées, avec des maisons d'enseignement depuis le niveau primaire jusqu'au niveau universitaire, sont déjà fréquentes et bénéfiques. Elles permettent aux gens, dès leur plus jeune âge, de s'approprier leur culture. Ces mises en commun sont toutefois plutôt ponctuelles et de courte durée, car elles monopolisent souvent les ressources limitées à une ou deux personnes du service d'archives et se déroulent dans des espaces restreints, faute de mieux. II faut dès lors envisager des partenariats et mécanismes incitatifs pour stimuler ces collaborations qui, selon certaines règles, pourraient participer à la formation de la maind'œuvre. De telles initiatives devraient être évaluées, non pas sur la base des revenus générés, mais sur l'offre culturelle offerte au public et l'atteinte d'objectifs en matière de diffusion et de formation. Il serait aussi souhaitable d'avoir une offre de partenariat provenant des institutions de formation de la relève archivistique dans des activités qui aillent au-delà du stage académique de quelques semaines. II peut s'agir de programmes de stages professionnels rémunérés. Ces stages permettraient à de jeunes diplômés de vivre une expérience de travail de quelques mois tout en étant rattachés à leur institution de formation, de pouvoir bénéficier des conseils et de l'expertise du corps professoral, $d$ 'avoir accès à des équipements et des outils de travail permettant de réaliser des projets et de faciliter leur accès au marché du travail. Pour l'employeur, cela lui permet d'atteindre des objectifs plus ambitieux, de bénéficier de l'expertise d'une main d'œuvre pour une plus longue 
période, d'initier du personnel dynamique au monde du patrimoine, de contribuer à augmenter les connaissances sur les archives et de participer à sensibiliser le personnel au patrimoine.

Une autre action envisageable au niveau de la participation citoyenne serait un incitatif permettant aux gens ayant atteint l'âge de la retraite de poursuivre un travail sur d'autres bases que le travail hebdomadaire à temps plein. On peut penser à des personnes provenant du monde de l'éducation, des communications, de la recherche qui, tout en restant actives, participent à la formation de la relève dans un centre d'archives, contribuent au travail de description des archives, à leur informatisation, à l'animation et aux visites de groupe.

\subsection{Une solution intégrée: les centres régionaux}

Pour sauvegarder leurs archives religieuses, les communautés ont tenté des partenariats avec des organismes civils, publics ou privés de leur région. Elles ont aussi souhaité s'associer avec leur diocèse, lorsque ce dernier est en faveur d'accueillir et d'héberger leurs archives. À la connaissance du RAR, des tentatives ont été faites dans certains diocèses, dont ceux de Chicoutimi, Québec et Montréal. Malheureusement, les commentaires reçus par le RAR font tous mention des mêmes problématiques: manque d'espace adéquat et manque de financement.

Comme exposé précédemment, une autre option proposée par le RAR consiste en ce que les communautés religieuses d'une même région prennent l'initiative de créer elles-mêmes un centre régional $d^{\prime}$ archives religieuses pour conserver et diffuser leurs archives. Cette mise en commun des ressources et la gestion intégrée du patrimoine permettraient d'avoir une vue d'ensemble des besoins et des solutions à mettre en place. La création de centres patrimoniaux religieux régionaux a aussi comme objectif de créer des pôles historiques, sociaux et culturels, permettant de:

- Maintenir le patrimoine dans sa région d'origine afin qu'il conserve tout son sens, à travers les mémoires personnelles et collectives;

- Entretenir ce patrimoine vivant, facteur d'identité et d'intégration, pour la génération actuelle et les futures; 
- Accroître la signification de ce patrimoine grâce à la mise en commun de l'expertise des professionnels du milieu et de la collaboration interdisciplinaire;

- Mettre en valeur ce patrimoine grâce à des initiatives visant à répondre à différents besoins et à des publics variés;

- Faciliter l'accès au patrimoine en des lieux uniques, que ce soit pour la population locale, les institutions d'enseignement, les touristes, etc.

La création de nouveaux pôles multidisciplinaires est primordiale pour stimuler l'intérêt des citoyens et donner un nouvel élan à l'éducation culturelle et artistique. Les centres de patrimoine religieux régionaux pourraient être non seulement des lieux de conservation, mais aussi des lieux de recherche, de diffusion et d'éducation. De plus, aider les centres régionaux de patrimoine religieux favoriserait le développement, à l'intérieur de ces centres, de musées et autres moyens de diffusion récréatifs. Ces moyens de diffusion pourraient être des attraits invitants pour les écoles et les familles, donnant un nouvel élan à l'éducation culturelle et soutenant le potentiel créatif des jeunes, leur sensibilisation et engagement en faveur de la culture. À travers des activités variées, les piliers de la culture que sont le loisir et l'éducation s'unissent. Le Musée des Hospitalières de l'Hôtel-Dieu de Montréal est un modèle déjà établi et inspirant pour représenter ce concept. II offre notamment des programmes éducatifs pour les élèves du primaire et du secondaire alliant visite du musée à des ateliers ${ }^{6}$ (ex. visites commentées du jardin jumelées à des ateliers d'apiculture ${ }^{7}$ ). En rendant le patrimoine vivant, facteur d'identité et d'intégration, les générations actuelles et futures seront responsabilisées envers leur propre héritage et leur culture.

La centralisation régionale avantage les citoyens, surtout lorsque I'implantation des institutions culturelles se fait dans des lieux centraux, faciles d'accès. Ces lieux pourraient être près des établissements scolaires et des secteurs touristiques. Cette solution est un outil de visibilité, par la création d'un pôle attractif où plusieurs activités de diffusion et d'éducation peuvent être mises en place dans un même lieu, facilitant ainsi les efforts de mise en valeur et donnant aux Québécois un accès privilégié à leur culture.

Comment trouver des lieux pour ces centres régionaux? Le Québec compte déjà un nombre important de bâtiments patrimoniaux. II serait 
intéressant de favoriser et d'encourager la mise en valeur de ces bâtiments avec des fonctions culturelles, dont ceux de services d'archives. Les centres régionaux de patrimoine religieux pourraient être les organismes par excellence pour s'établir dans de tels lieux patrimoniaux. En effet, ces centres s'inscrivent parfaitement dans un contexte d'aménagement du territoire qui favorise la conservation du patrimoine, l'archéologie, l'urbanisme et la qualité architecturale des lieux. Par exemple, I'implantation du Centre du patrimoine religieux à Montréal dans un site patrimonial religieux s'inscrirait admirablement dans sa mission de mise en valeur et de diffusion. Elle permettrait de conserver et de mettre en valeur la signification et la portée du lieu, grâce à la préservation des liens existant entre le contenant (bâtiment) et son contenu (archives, publications, biens mobiliers et œuvres d'art). II serait alors possible d'aller au-delà de la conservation du patrimoine bâti, en protégeant la mémoire des lieux.

Le centre régional $d$ 'archives religieuses le plus avancé dans sa réalisation est le Centre d'archives Mgr-Antoine-Racine, aménagé dans la cathédrale Saint-Michel à Sherbrooke. De plus petite envergure que le centre qui sera nécessaire pour la région de Montréal, ce centre d'archives rassemblera, les archives du Séminaire de Sherbrooke et celles de l'Archevêché. Vers la fin du projet, trois communautés religieuses ayant marqué le développement de la région rejoindront le centre. II s'agit d'une mise en valeur exceptionnelle de plusieurs patrimoines réunis: historique, architectural, artistique et archivistique. Les travaux sont évalués à $1281604 \$$ et le Centre a reçu une subvention gouvernementale de 606660 \$ en vertu du programme Aide aux immobilisations. Le reste du financement provient des partenaires privés.

Somme toute, l'établissement de centres régionaux de patrimoine religieux est une solution qui permettrait de centraliser les interventions gouvernementales par région, facilitant ainsi la prise de décisions relatives à la conservation et à la diffusion du patrimoine religieux.

\section{CONCLUSION}

Les patrimoines religieux archivistique, documentaire et muséal font partie intégrante du patrimoine du Québec et il est important qu'ils soient reconnus comme tels par la société et l'État. Ils témoignent notamment des origines des systèmes d'éducation, hospitaliers et services sociaux ainsi que 
de divers événements qui ont marqué l'histoire du Québec. Ils sont aussi des témoins éloquents des liens historiques qu'entretient le Québec avec les autres nations de la Francophonie, et peuvent être un outil facilitant la préservation de la langue française. L'histoire des missions religieuses locales et à l'étranger, telle que documentée dans les archives, reflètent la diversité culturelle du Québec et, pour les personnes issues de l'immigration, peuvent favoriser leurs participation, intégration et compréhension de la culture de leur nouvelle terre d'accueil. Les autochtones peuvent également trouver, dans les archives religieuses, du matériel leur permettant de se réapproprier leurs langues ancestrales.

Malheureusement, les communautés religieuses sont majoritairement en fin de vie. Pour plusieurs, leur patrimoine est en situation de précarité. Des solutions sont déjà explorées par le milieu religieux afin d'assurer la préservation et l'accessibilité à ce patrimoine. La concertation entre les intervenants est déjà engagée, mais elle ne saurait mener à des solutions pérennes sans un soutien logistique et financier pour développer, implanter et veiller à la viabilité à long terme de ces projets. Ce mémoire recommande des incitatifs, des partenariats et autres projets, pour lesquels une aide financière aurait un net impact sur la mise en valeur du patrimoine religieux et de la culture québécoise. Que l'on parle de partenariats avec les municipalités, d'aménagement du territoire favorisant la conservation du patrimoine, d'un nouvel élan à l'éducation culturelle ou d'un engagement revitalisé en faveur de la culture québécoise, on ne peut ignorer ce que les archives religieuses peuvent contribuer. En définitive, il nous paraît impossible d'imaginer une politique culturelle renouvelée pour le Québec sans imaginer de nouveaux efforts pour la conservation et la mise en valeur des archives religieuses.

\section{NOTES}

1. Conférence des évêques catholiques du Canada. (2016). Histoire de l'Église au Canada. Repéré à http://www.cccb.ca/site/frc/eglise-au-canada-et-ailleurs/leglise-catholiqueau-canada/histoire-de-leglise-au-canada

2. Église catholique en France. Glossaire. Repéré à http://www.eglise.catholique.fr/ glossaire/

3. Sondage de la TCARM, mai 2016. 
4. Gilles Héon. L'avenir du patrimoine archivistique religieux du Québec. Mémoire de l'Association des archivistes du Québec en vue des auditions publiques de la Commission de la culture dans le cadre de la consultation générale sur le patrimoine religieux, $1^{\text {er }}$ septembre 2005, paru dans Archives, volume 37, no. 2, 2005-2006, p. 176-177.

5. Observatoire de la culture et des communications du Québec. État des lieux du patrimoine des institutions muséales et des archives. Cahier 6. Les archives au Québec, des ressources documentaires à découvrir, 2006, 79 pages. Repéré à http://www.stat. gouv.qc.ca/statistiques/culture/patrimoine-musees-archives/cahier-06-etatdeslieux.pdf

6. Programmes éducatifs du Musée des Hospitalières de l'Hôtel-Dieu de Montréal. Repéré à http://museedeshospitalieres.qc.ca/programmes-educatifs/

7. Visites du jardin des Hospitalières. Repéré à http://museedeshospitalieres.qc.ca/activity/ visites-commentees-du-jardin-du-monastere/ 